\title{
Three new species of Cucullanus (Nematoda: Cucullanidae) from marine fishes off New Caledonia, with a key to species of Cucullanus from Anguilliformes
}

\author{
František Moravec ${ }^{1}$ and Jean-Lou Justine ${ }^{2}$,* \\ ${ }^{1}$ Institute of Parasitology, Biology Centre of the Czech Academy of Sciences, Branišovská 31, 37005 České Budějovice, \\ Czech Republic \\ ${ }^{2}$ Institut Systématique, Évolution, Biodiversité (ISYEB), Muséum national d'Histoire naturelle, CNRS, Sorbonne Université, \\ EPHE, CP 51, 57 rue Cuvier, 75005 Paris, France
}

Received 16 July 2018, Accepted 8 August 2018, Published online 20 September 2018

\begin{abstract}
Based on light and scanning electron microscopical studies of nematode specimens from the digestive tract of some rarely collected anguilliform and perciform fishes off New Caledonia, three new species of Cucullanus Müller, 1777 (Cucullanidae) are described: C. austropacificus n. sp. from the longfin African conger Conger cinereus (Congridae), C. gymnothoracis n. sp. from the lipspot moray Gymnothorax chilospilus (Muraenidae), and C. incognitus $\mathrm{n}$. sp. from the seabream Dentex fourmanoiri (Sparidae). Cucullanus austropacificus $\mathrm{n}$. $\mathrm{sp}$. is characterized by the presence of cervical alae, ventral sucker, alate spicules 1.30-1.65 mm long, conspicuous outgrowths of the anterior and posterior cloacal lips and by elongate-oval eggs measuring $89-108 \times 48-57 \mu \mathrm{m}$; C. gymnothoracis $\mathrm{n}$. $\mathrm{sp}$. is similar to the foregoing species, but differs from it in the absence of cervical alae and the posterior cloacal outgrowth, in the shape and size of the anterior cloacal outgrowth and somewhat shorter spicules $1.12 \mathrm{~mm}$ long; C. incognitus $\mathrm{n}$. sp. (based on female morphology) differs from other congeneric species parasitic in the Sparidae mainly in possessing cervical alae, the postequatorial vulva, phasmids situated at the mid-length of the tail and in the size of the eggs $(75-84 \times 45-66 \mu \mathrm{m})$. A key to species of Cucullanus parasitizing anguilliform fishes is provided.
\end{abstract}

Key words: Parasitic nematode, taxonomy, Seuratoidea, Osteichthyes, Conger, Gymnothorax, Dentex, South Pacific.

Résumé - Trois nouvelles espèces de Cucullanus (Nematoda: Cucullanidae) de poissons marins au large de la Nouvelle-Calédonie, avec une clé des espèces de Cucullanus d'Anguilliformes. Sur la base d'une étude en microscopie photonique et électronique à balayage de spécimens de nématodes provenant du tube digestif de quelques poissons anguilliformes et perciformes rarement pêchés en Nouvelle-Calédonie, trois nouvelles espèces de Cucullanus Müller, 1777 (Cucullanidae) sont décrites: C. austropacificus n. sp. de Conger cinereus (Congridae), C. gymnothoracis n. sp. de Gymnothorax chilospilus (Muraenidae) et C. incognitus n. sp. de Dentex fourmanoiri (Sparidae). Cucullanus austropacificus n. sp. est caractérisé par la présence d'ailes cervicales, une ventouse ventrale, des spicules ailés de 1,30-1,65 $\mathrm{mm}$ de long, des excroissances remarquables des lèvres cloacales antérieure et postérieure et d'oeufs ovales et allongés mesurant 89-108 $\times$ 48-57 $\mu \mathrm{m}$. C. gymnothoracis $\mathrm{n}$. sp. est semblable à l'espèce précédente, mais en diffère en l'absence d'ailes cervicales et d'excroissance cloacale postérieure, par la forme et la taille de l'excroissance cloacale antérieure et par des spicules un peu plus courts de $1,12 \mathrm{~mm}$. C. incognitus n. sp. (basé sur la morphologie de la femelle) diffère des autres espèces congénères parasites chez les Sparidae principalement par la possession d'ailes cervicales, une vulve postéquatoriale, des phasmides situés à mi-longueur de la queue et par la taille des oeufs $(75-84 \times 45-66 \mu \mathrm{m})$. Une clé des espèces de Cucullanus parasitant les poissons anguilliformes est fournie.

The nematode genus Cucullanus Müller, 1777 (Cucullanidae) contains a large number of species parasitizing freshwater, brackish-water or marine fishes around the world; more rarely they are found in aquatic turtles $[15,27,35]$. Because of their rather uniform morphology and the inadequate descriptions of many nominal species, it is practically impossible to

\footnotetext{
*Corresponding author: moravec@paru.cas.cz
}

make a detailed comparison between all of them. Consequently, some authors prefer to deal with these parasites according to their host groups $[12,30,35]$ or their zoogeographical region $[9,26,44]$.

Only the following three nominal species of Cucullanus, all parasites of marine fishes, have been recorded from off New Caledonia: C. bourdini Petter \& Le Bel, 1992 from Aprion virescens Valenciennes, Lutjanus gibbus (Forsskål), 
Pristipomoides auricilla (Jordan, Evermann \& Tanaka) and P. filamentosus (Valenciennes) (all Lutjanidae); C. bulbosus (Lane, 1916) from Carangoides fulvoguttatus (Forsskål) (Carangidae); and C. epinepheli Moravec \& Justine, 2017 from Epinephelus chlorostigma (Valenciennes) (Serranidae) [22-24, 36].

Parasitological examinations of some rarely collected marine fishes off New Caledonia conducted between 2009 and 2011 yielded, among other helminths, nematodes referable to Cucullanus from the digestive tract of Conger cinereus Rüppell (Congridae, Anguilliformes), Gymnothorax chilospilus Bleeker (Muraenidae, Anguilliformes), and Dentex fourmanoiri Akazaki \& Séret (Sparidae, Perciformes). These proved to represent three morphologically different, previously unknown species of Cucullanus, which are described herein.

Whereas Co. cinereus and G. chilospilus are tropical, reefassociated fishes widespread in the Indo-Pacific region, D. fourmanoiri is a rare, deep-water fish with a limited distribution in the Southwest Pacific, occurring near the Chesterfield Islands and New Caledonia [11].

\section{Materials and methods}

Fish were caught off New Caledonia by various, and sometimes unusual, means. The seabream Dentex fourmanoiri was caught by line; the conger Conger cinereus was taken in a cage baited for the collection of Nautilus; and the moray Gymnothorax chilospilus was obtained from a New Caledonian sea krait, Laticauda saintgironsi Cogger \& Heatwole, collected on a small islet, Ilôt Amédée, off Nouméa, New Caledonia. As this host is an emblematic protected species, an indirect sampling method without any effect on survival was used [5]: a gentle massage of the sea krait abdomen provided the stomach content by regurgitation, and the regurgitated contents included the moray eel. Parasites were obtained by a wash method [14]. The nematodes for morphological studies were fixed in hot $4 \%$ formalin or $70 \%$ ethanol. For light microscopical examination (LM), they were cleared with glycerine. Drawings were made with the aid of a Zeiss microscope drawing attachment. Specimens used for scanning electron microscopical examination (SEM) were postfixed in $1 \%$ osmium tetroxide (in phosphate buffer), dehydrated through a graded acetone series, critical-point-dried and sputter-coated with gold; they were examined using a JEOL JSM-7401F scanning electron microscope at an accelerating voltage of $4 \mathrm{kV}$ (GB low mode). All measurements are in micrometres, unless otherwise indicated. The fish nomenclature adopted follows FishBase [11].

\section{Results}

\section{Cucullanidae Cobbold, 1864}

Cucullanus austropacificus n. sp. Figures 1-3

urn:1sid:zoobank.org:act:4F1D1180-8FE8-498F-A38C2C63A4C9A36E

Type host: Longfin African conger Conger cinereus Rüppell (Congridae, Anguilliformes).

Site of infection: Digestive tract.
Type locality: Deep sea, $400 \mathrm{~m}$ depth, near Passe de Dumbéa, off Nouméa, New Caledonia (collected 3 July 2009).

Prevalence and intensity: 1 fish infected/1 fish examined; 12 nematodes.

Deposition of type specimens: Muséum national d'Histoire naturelle, Paris, France (male holotype, female allotype and 3 paratypes [2 males and 1 female], MNHN JNC 2993); Helminthological Collection, Institute of Parasitology, Biology Centre of the Czech Academy of Sciences, České Budějovice, Czech Republic (3 paratypes [2 males and 1 female], Cat. No. N-1167).

Etymology: The specific name of this nematode is a Latin adjective composed of the words australis (= southern) and Pacificus (= Pacific), which relates to the region of the occurrence of this parasite, that is South Pacific.

\section{Description}

General: Medium-sized nematodes. Body whitish, elongate, somewhat narrowed in region between posterior end of pseudobuccal capsule and posterior end of oesophagus (Figs. 1A and 1B). Narrow lateral cervical alae present, beginning approximately at level of posterior end of pseudobuccal capsule and extending posteriorly to short distance anterior to posterior end of oesophagus (Figs. 1A, 3A and 3B). Cephalic end slightly asymmetrical in lateral view (Figs. 1B, $1 \mathrm{C}$ and 2A). Oral aperture dorsoventrally elongate, surrounded by raised narrow membranous ala (collarette) supported by row of c. 100 minute basal teeth (Figs. 1C, 2A, and 2B). Four submedian cephalic double papillae and pair of lateral amphids present (Figs. 1C and 2A). Oesophagus muscular, expanded at anterior end to form bulbous pseudobuccal capsule (oesophastome); posterior part of oesophagus also expanded, somewhat narrower than oesophastome in lateral view (Figs. 1A and 1B). Oesophagus opens into intestine through large valve. Nerve ring encircles oesophagus at distance representing 29\%-35\% of oesophageal length. Deirids small, situated in posterior half of distance between nerve ring and posterior end of oesophagus (Figs. 1A, 1B, 2C, 3A, 3B). Postdeirids not found. Excretory pore in region of oesophagointestinal junction (Fig. 1B). Tail of both sexes conical, sharply pointed at tip.

Male ( 7 specimens; measurements of holotype in parentheses): Length of body 15.80-26.15 (26.15) mm, maximum width 422-680 (680); width at level of oesophastome 286490 (490), at middle of oesophagus 218-408 (408). Maximum width of cervical alae 18-27 (24). Length of entire oesophagus 1.59-2.31 (2.31) mm, representing 9-11 (9)\% of whole body length; length of oesophastome 340-530 (503), its width 272-408 (394); minimum width of oesophagus 95-150 (150); maximum width of posterior part of oesophagus 217299 (299). Distance of nerve ring from anterior extremity 503-789 (789), representing 29-35 (34)\% of oesophageal length. Deirids and excretory pore $1.02-2.12(2.12) \mathrm{mm}$ and 1.32-2.57 (2.01) mm, respectively, from anterior end of body. Posterior end of body curved ventrally. Ventral sucker and ventral precloacal oblique muscle bands present (Figs. 1E, 3C). Cloacal region somewhat elevated. Large median papillalike formation present anterior to cloacal opening, being 


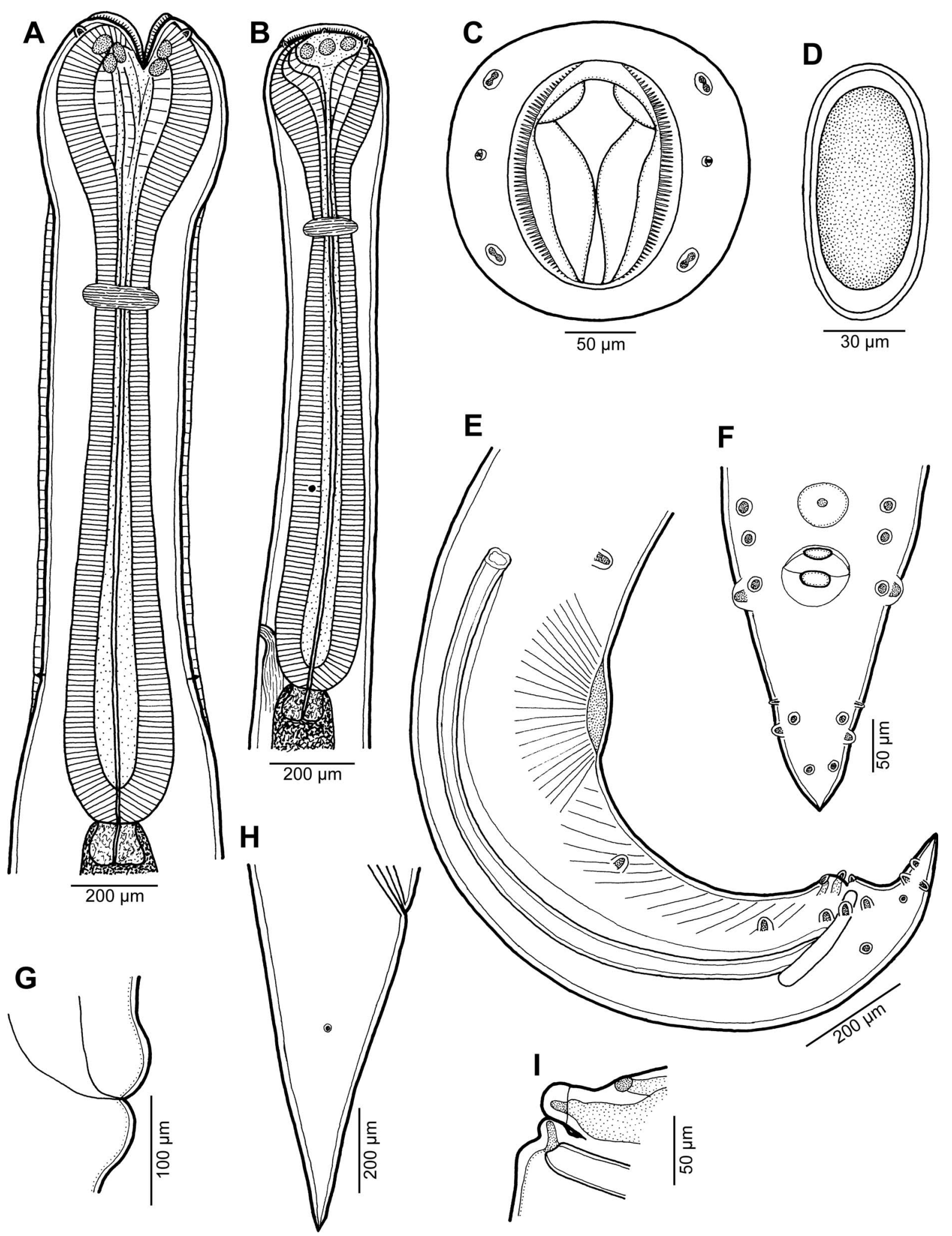

Figure 1. Cucullanus austropacificus n. sp. from Conger cinereus. (A) Anterior end of large male (body length $24 \mathrm{~mm}$ ), dorsoventral view; (B) anterior end of small male (body length $17 \mathrm{~mm}$ ), lateral view; (C) cephalic end, apical view; (D) fully developed egg; (E) posterior end of male, lateral view; (F) tail of male, ventral view; (G) vulva, lateral view; (H) tail of female, lateral view; (I) region of cloaca, lateral view. 

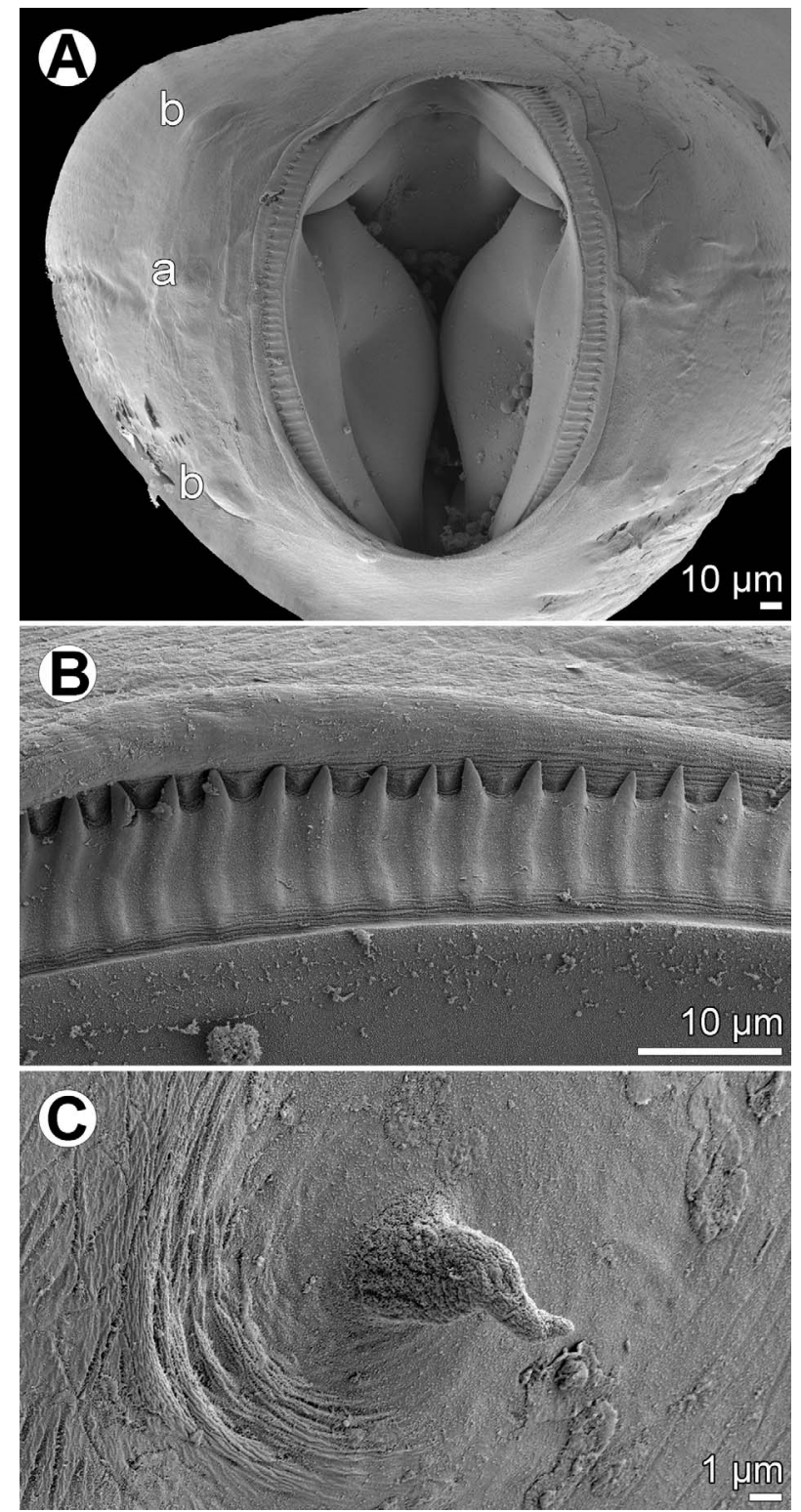

Figure 2. Cucullanus austropacificus n. sp., scanning electron micrographs. (A) Cephalic end, apical view; (B) cephalic teeth; (C) deirid. (a) amphid; (b) cephalic double papilla.

accompanied by conspicuously elevated transversely-oval median outgrowth adherent to inner rim of anterior cloacal lip; posterior cloacal lip with median, transversely-oval, conspicuously elevated outgrowth at tip (Figs. 1E, 1F, 1I, 3C-3F). Spicules equal, 1.30-1.65 (1.59) mm long, representing 5-10 (6)\% of body length. Gubernaculum well sclerotized, rod-like in lateral view, 225-291 (291) long (Fig. 1E). Caudal papillae 11 pairs: 5 pairs of subventral preanal papillae, 2 pairs of adanal papillae (1 subventral and 1 lateral) and 4 pairs of postanal papillae (2 subventral, 1 lateral and 1 dorsolateral); subventral pairs of postanals in second half of tail; postanal pair of laterals (representing phasmids) slightly anterior to level of first subventral pair; papillae of dorsolateral postanal pair slightly anterior to level of last pair of subventrals (Figs. 1E, 1F, 3C-3E). Length of tail 219-462 (462) (Figs. 1E, 1F, 3C-3E).
Female (5 ovigerous specimens; measurements of allotype in parentheses): Length of body 26.43-34.61 (34.61) mm, maximum width 680-734 (734); width at level of oesophastome 476-558 (558), at middle of oesophagus 408-422 (408). Maximum width of cervical alae 24-33 (33). Length of entire oesophagus 2.42-2.58 (2.58), representing 7-9 (7)\% of whole body length; length of oesophastome 544-625 (612), its width 422-462 (462); minimum width of oesophagus 109-163 (109); maximum width of posterior part of oesophagus 272-326 (326). Distance of nerve ring from anterior extremity 789-857 (857), representing 32-33 (33)\% of oesophageal length. Deirids and excretory pore 1.84-1.97 (1.96) and 2.34-2.77 (2.77) mm, respectively, from anterior end of body. Vulva postequatorial, 15.89-20.84 (20.84) $\mathrm{mm}$ from anterior extremity, at 60-63 (60)\% of body length; vulval lips slightly elevated (Fig. 1G). Vagina directed anteriorly from vulva. Uteri opposed. Fully developed eggs elongate-oval, thin-walled, size 84-108 × 48-57 $(93-99 \times 54-57)$, with uncleaved contents (Fig. 1D). Length of tail 666-857 (694); phasmids situated approximately at its middle (Fig. 1H).

\section{Remarks}

To date, the following 12 species of Cucullanus are known to occur in anguilliform fishes: C. anguillae Wang \& Ling, 1975 from Anguilla japonica (Temminck \& Schlegel) in China [45]; C. australiensis Baylis, 1927 (syn. C. faliexae Morand \& Rigby, 1998) from Gymnothorax cf. pictus (Ahl) and G. javanicus (Bleeker) from off Australia and French Polynesia, respectively [2, 18]; C. egyptae Abdel-Ghaffar, Bashtar, Abdel-Gaber, Morsy, Mehlhorn, Al Quraishy \& Mohammed, 2014 [species inquirenda; 31] from Anguilla anguilla (Linnaeus) in Egypt [1]; C. hainanensis Xu, Zhang \& Li, 2014 from Muraenichthys gymnopterus (Bleeker) in the South China Sea [41]; C. hians (Dujardin, 1845) [syn. C. praecinctus (Dujardin, 1845)] mainly from Conger conger (Linnaeus) off the Atlantic coasts of Europe and Africa [8, 10, 37]; C. muraenesocis Yamaguti, 1961 from Muraenesox cinereus (Forsskål) off Japan [47]; C. murenophidis Campana-Rouget, 1957 from Muraena robusta Osório off the Atlantic coast of Africa [7]; C. oceaniensis Moravec, Sasal, Würtz \& Taraschewski, 2005 from Anguilla marmorata Quoy \& Gaimard and Anguilla cf. obscura Günther in Oceania [30]; C. pedroi Timi \& Lanfranchi, 2006 from Conger orbignianus Valenciennes off the Atlantic coast of Argentina and Brazil [40, 44]; $C$. robustus Yamaguti, 1935 (syn. C. filiformis Yamaguti, 1935) from Conger myriaster (Brevoort) from off Japan and the Korean Peninsula [32, 46]; C. truttae Fabricius, 1794 from Anguilla anguilla (but mostly parasitic in freshwater salmonids and cyclostomes) in Europe [20, 21]; and $C$. wangi Xu, Zhang \& Li, 2014 (syn. Indocucullanus muraenesocis Yin \& Zhang, 1983) from Muraenesox cinereus off China [41, 48].

Cucullanus austropacificus $\mathrm{n}$. sp. differs from all of the above-mentioned species, except for $C$. truttae, in the presence of cervical alae, but also in some other morphological features. By the structure of the cloacal region, the new species is most similar to $C$. pedroi parasitizing congeneric fish host in the western Atlantic Ocean, but differs from it in the 


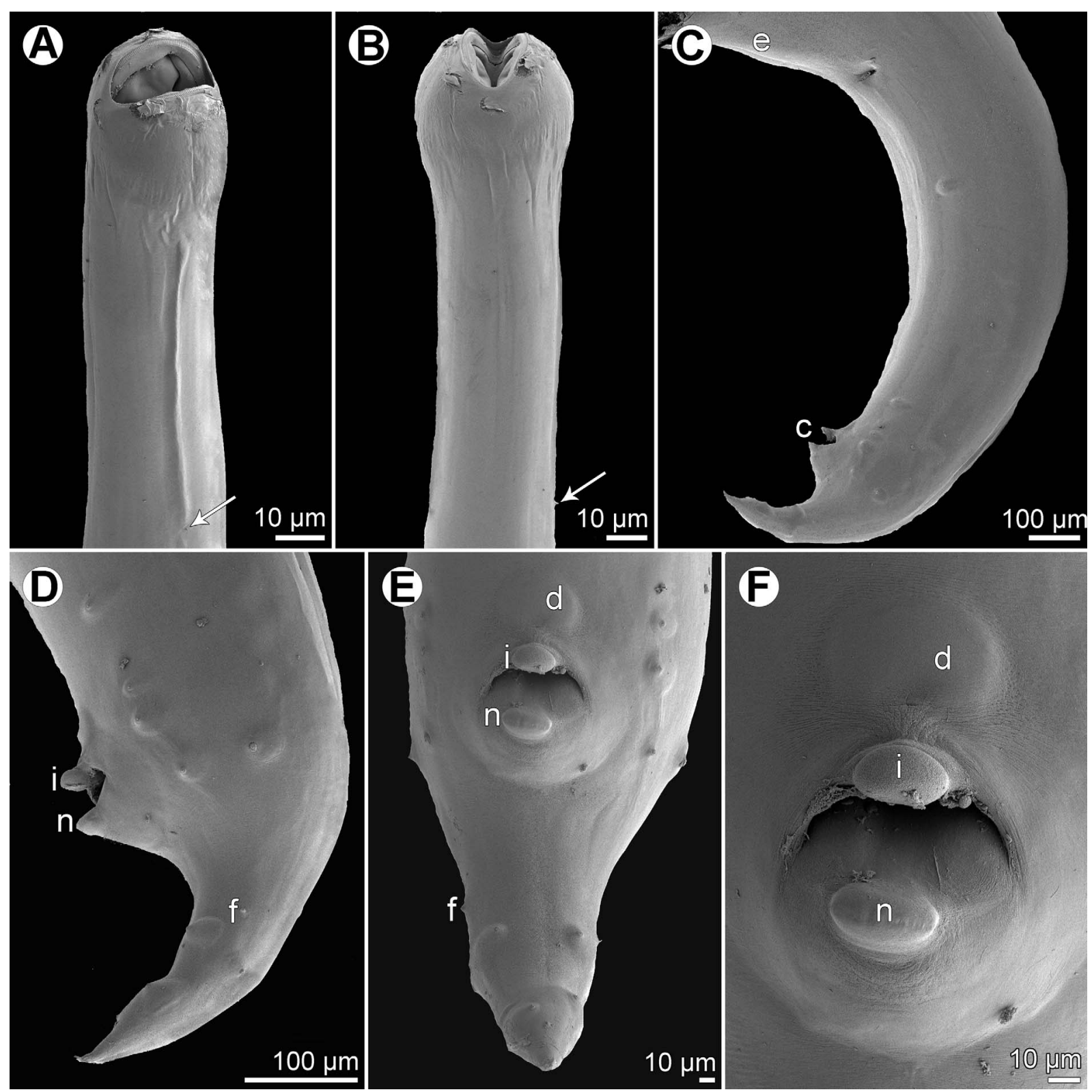

Figure 3. Cucullanus austropacificus n. sp., scanning electron micrographs. (A, B) Anterior end of body, sublateral and dorsoventral views, respectively (arrows indicate deirids; note presence of cervical alae); (C) posterior end of male, lateral view; (D, E) tail of male, lateral and ventral views, respectively; (F) region of cloaca, ventral view (higher magnification). (c) cloaca; (d) median precloacal papilla-like formation; (e) ventral sucker; (f) phasmid; (i) median outgrowth of anterior cloacal lip; (n) median outgrowth of posterior cloacal lip.

shape (more elongate in $C$. pedroi) of the oesophastome, its anterior cloacal outgrowth is smaller than the posterior outgrowth ( $v s$. anterior outgrowth larger than the posterior one), the lower posterior part of the posterior cloacal lip is without denticulations ( $v s$. denticulations present) and the sixth pair of subventral papillae is situated posterior to the cloaca (vs. at the level of the cloaca) (see Figs. 60-62 in Vieira et al. [44]). The distinction of C. austropacificus n. sp. from other congeners parasitizing anguilliform fishes is more apparent from the key at the end of the Discussion in this article.
In the same individual conger, we also collected digeneans, including Acaenodera nautili Bray \& Justine, 2011, and larvae of the trypanorhynch cestode Microbothriorhynchus coelorhynchi Yamaguti, 1952 [3, 4].

\section{Cucullanus gymnothoracis n. sp. Figures 4-6}

urn:1sid:zoobank.org:act:D5E65F49-66D2-466C-BC485EC0CC88E1F9

Type host: Lipspot moray Gymnothorax chilospilus Bleeker (Muraenidae, Anguilliformes), obtained from the stomach 


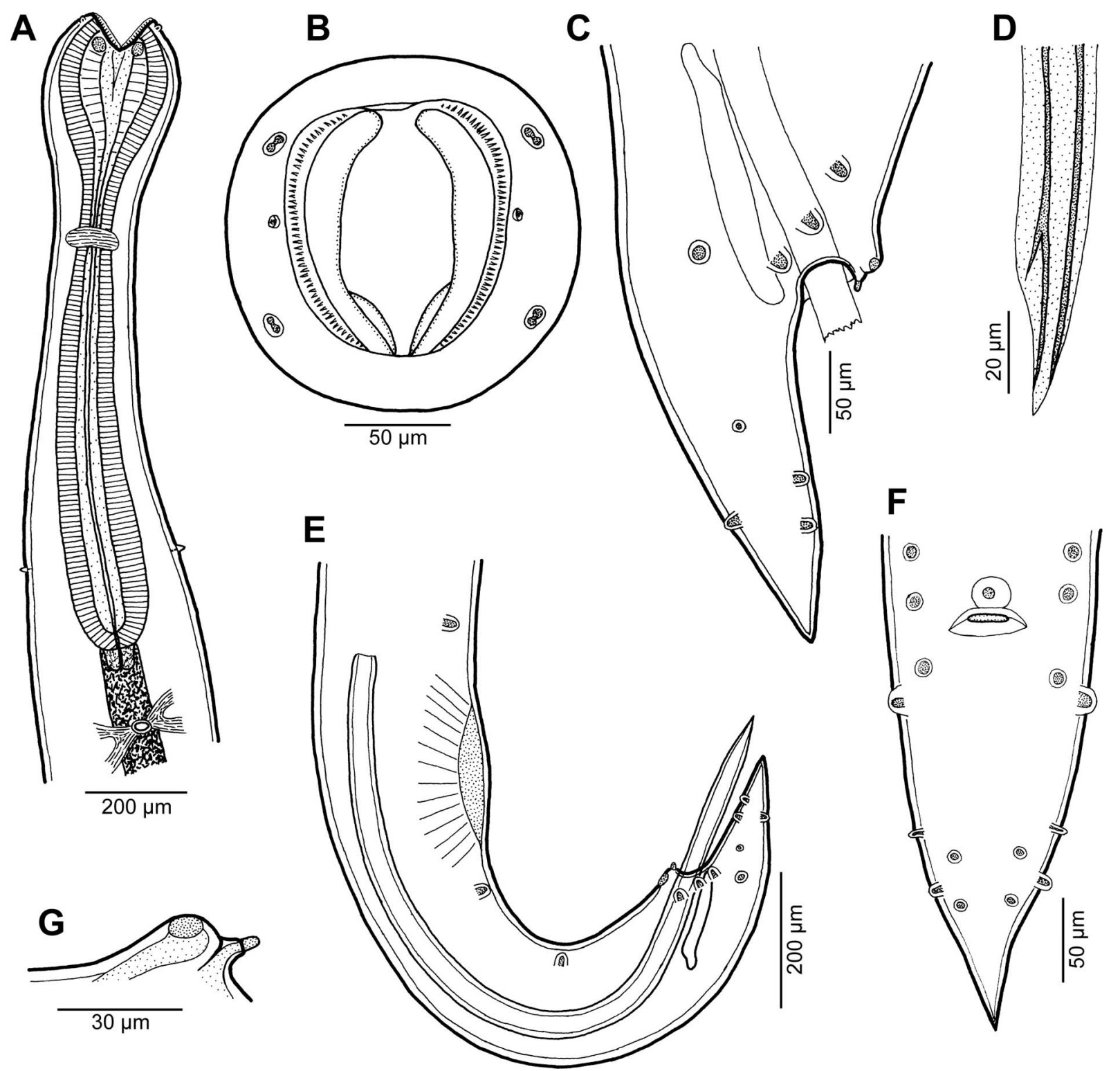

Figure 4. Cucullanus gymnothoracis n. sp. from Gymnothorax chilospilus, male. (A) Anterior end of body, ventral view; (B) cephalic end, apical view; (C) tail, lateral view; (D) distal end of spicule, lateral view; (E) posterior end of body, lateral view; (F) tail, ventral view; (G) median precloacal papilla-like formation and outgrowth of anterior cloacal lip, lateral view.

content of a male New Caledonian sea krait, Laticauda saintgironsi Cogger \& Heatwole, 2006 (see Materials and methods section).

Site of infection: Digestive tract.

Type locality: Near Ilôt Amédée, off Nouméa, New Caledonia (collected 13 February 2011) (parasitological number MNHN JNB 004).

Prevalence and intensity: 1 fish infected/5 fish examined; 1 nematode.

Deposition of type specimen: Helminthological Collection, Institute of Parasitology, Biology Centre of the Czech Academy of Sciences, České Budějovice, Czech Republic (male holotype mounted on SEM stub, Cat. No. N-1168).

Etymology: The specific name of this nematode relates to the genitive form of the generic name of the host.

\section{Description}

Male (1 specimen, holotype): Medium-sized nematode. Body whitish, elongate, somewhat narrowed in region between posterior end of pseudobuccal capsule and posterior end of oesophagus (Fig. 4A). Cuticle slightly transversely striated (Figs. 5E, 6C, 6D). Length of body $14.63 \mathrm{~mm}$, maximum width 394; width at level of oesophastome 286, at middle of oesophagus 177. Lateral alae absent (Figs. 4A, 5E). Cephalic end somewhat asymmetrical in lateral view (Fig. 5A). Oral aperture dorsoventrally elongate, surrounded by raised narrow membranous ala (collarette) supported by row of $c .120$ minute basal teeth (Figs. 4B, 5A, 5C, 5D). Four submedian cephalic double papillae and pair of lateral amphids present (Figs. 4B, $5 \mathrm{~A}-5 \mathrm{C}$ ). Oesophagus muscular, expanded at anterior end to 


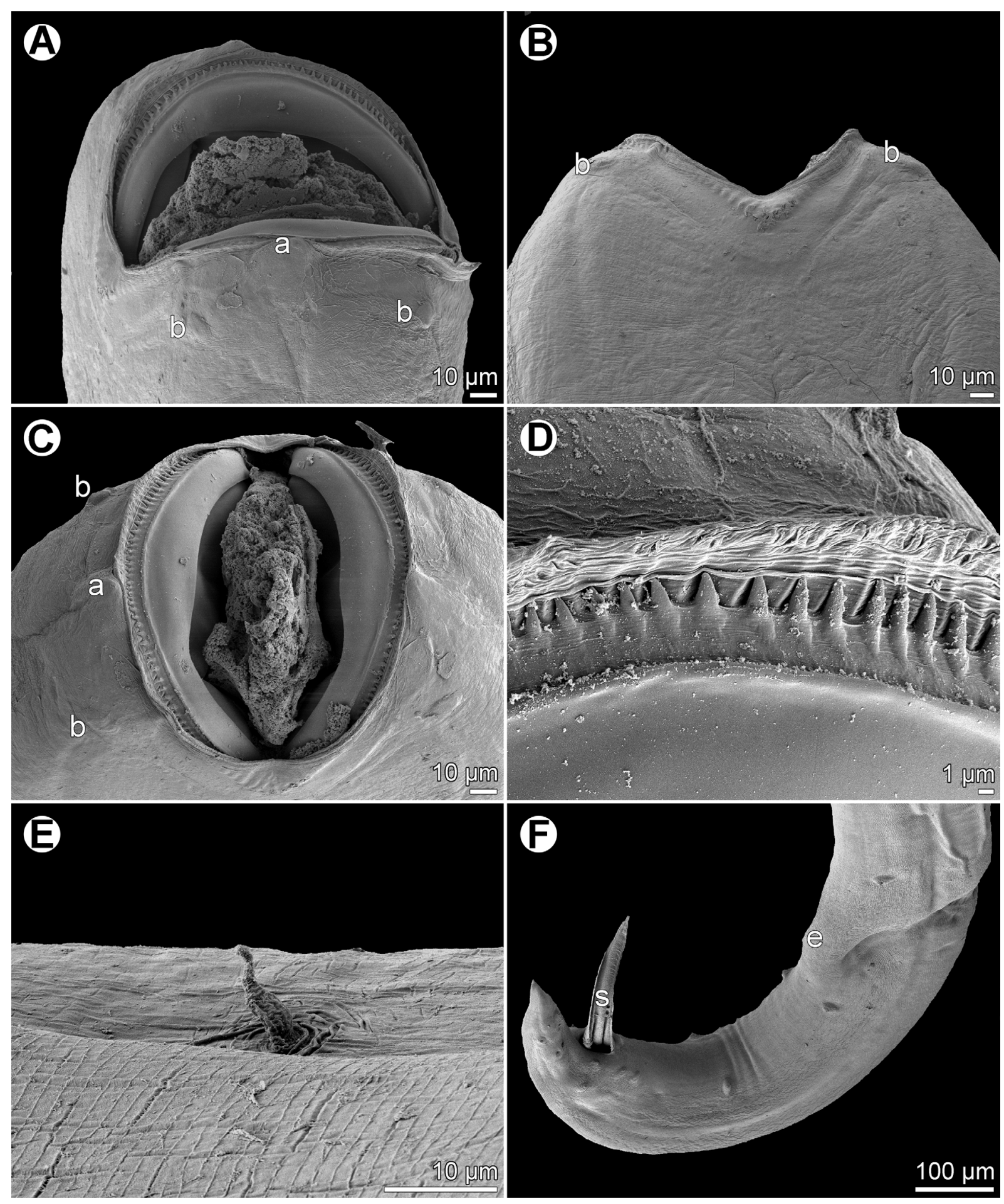

Figure 5. Cucullanus gymnothoracis n. sp., scanning electron micrographs of male. (A, B) Cephalic end, lateral and dorsoventral views, respectively; (C) same, apical view; (D) cephalic teeth; (E) deirid; (F) posterior end of body, lateral view. (a) amphid; (b) cephalic double papilla; (e) ventral sucker; (s) spicule.

form bulbous pseudobuccal capsule (oesophastome); posterior part of oesophagus also expanded, somewhat narrower than oesophastome in lateral view (Fig. 4A). Length of entire oesophagus $1.40 \mathrm{~mm}$, representing $9.6 \%$ of whole body length; length of oesophastome 367 , its width 258 ; minimum width of oesophagus 68; maximum width of posterior part of oesophagus 177. Oesophagus opens into intestine through large valve. Distance of nerve ring from anterior extremity 480 , representing $35 \%$ of oesophageal length. Deirids small, situated short distance anterior to posterior end of oesophagus (Figs. 4A, 5E). Postdeirids not found. Excretory pore situated at short distance posterior to oesophago-intestinal junction 

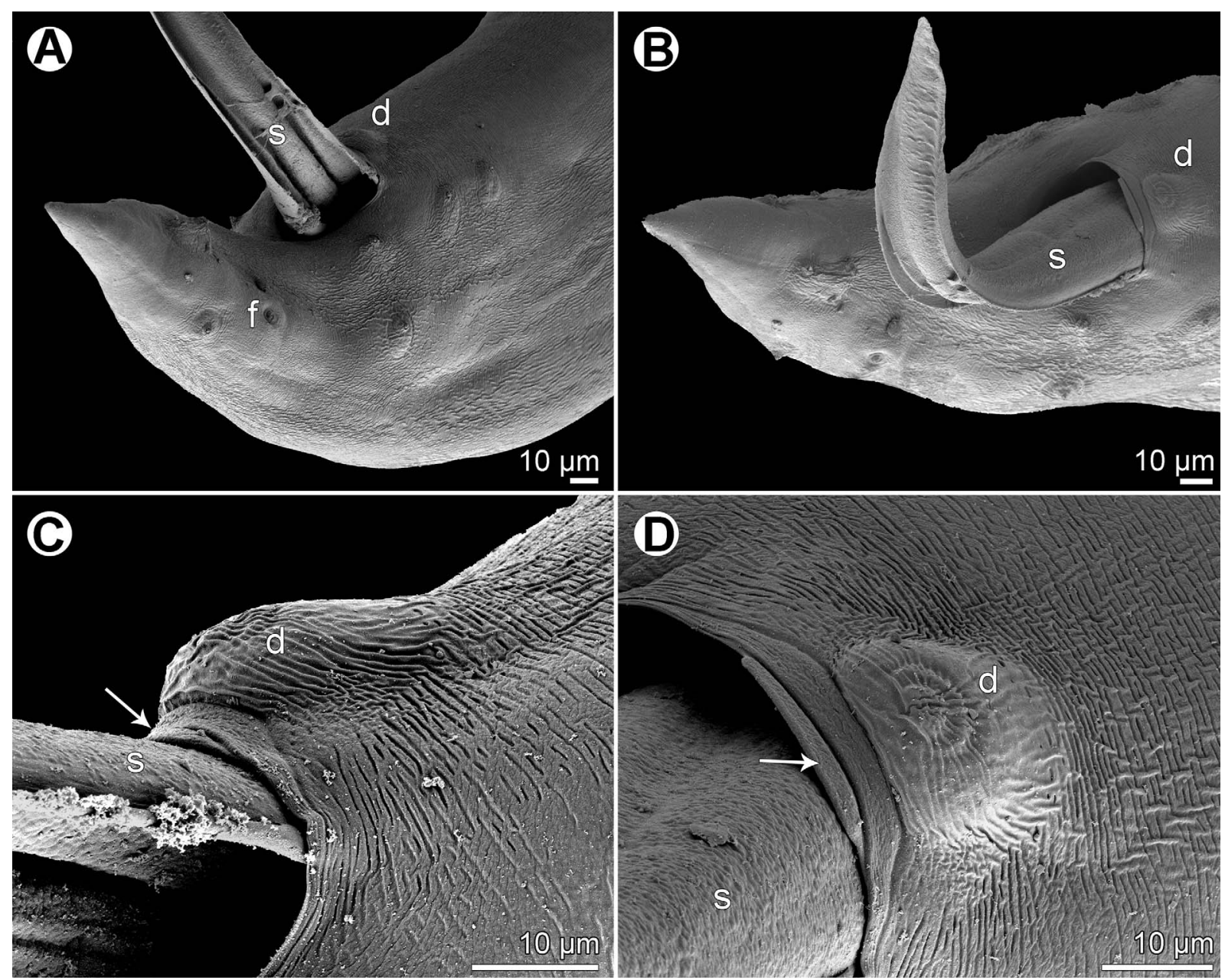

Figure 6. Cucullanus gymnothoracis n. sp., scanning electron micrographs of male. (A, B) Tail, lateral and ventral views, respectively; (C, D) precloacal region, lateral and ventral views, respectively (arrows indicate outgrowth of anterior cloacal lip). (d) median precloacal papilla-like formation; (f) phasmid; (s) spicule.

(Fig. 4A). Deirids and excretory pore $1.16 \mathrm{~mm}$ and $1.48 \mathrm{~mm}$, respectively, from anterior end of body. Posterior end of body curved ventrally. Ventral precloacal sucker present (Figs. 4E, 5F). Cloacal region not elevated. Large, somewhat elevated median papilla-like formation present anterior to cloacal opening, being accompanied by slightly elevated median transverse outgrowth adherent to inner rim of anterior cloacal lip (Figs. 4C, 4E-4G, 6A-6D). Spicules equal, alate, $1.12 \mathrm{~mm}$ long, with pointed posterior ends (Figs. 4D, 4E, 5F, 6B), representing $7.6 \%$ of body length. Gubernaculum well sclerotized, rod-like with narrow proximal part in lateral view, 201 long (Figs. 4C, 4E). Caudal papillae 11 pairs: 5 pairs of subventral preanal papillae, 2 pairs of adanal papillae (1 subventral and 1 lateral) and 4 pairs of postanal papillae (2 subventral, 1 lateral and 1 dorsolateral); subventral pairs of postanals in second half of tail; postanal pair of laterals (representing phasmids) slightly anterior to level of first subventral pair; papillae of dorsolateral postanal pair slightly anterior to level of last pair of subventrals (Figs. 4C, 4E, 4F, 5F, 6A, 6B). Tail conical, pointed, 245 long (Figs. 4C, 4E, 4F, 5F, 6A, 6B).
Female: Not known.

\section{Remarks}

The morphology and measurements of this nematode specimen, as well as the fact that it was collected from the congeneric fish host in the nearby region, show its similarity to C. australiensis, the species originally described by Baylis [2] from Gymnothorax cf. pictus off Australia. Later Morand \& Rigby [18] established C. faliexae from G. javanicus in French Polynesia, but it was subsequently synonymized with C. australiensis [30]. Cucullanus australiensis has not yet been studied by SEM, so its detailed morphology remains unknown. Nevertheless, the present specimen differs markedly from C. australiensis in the considerably more posterior situation of deirids and the excretory pore and, therefore, this is considered to represent a separate species. Comparison of C. gymnothoracis $\mathrm{n}$. sp. with other congeneric species is apparent from the key presented at the end of the Discussion in this article. 

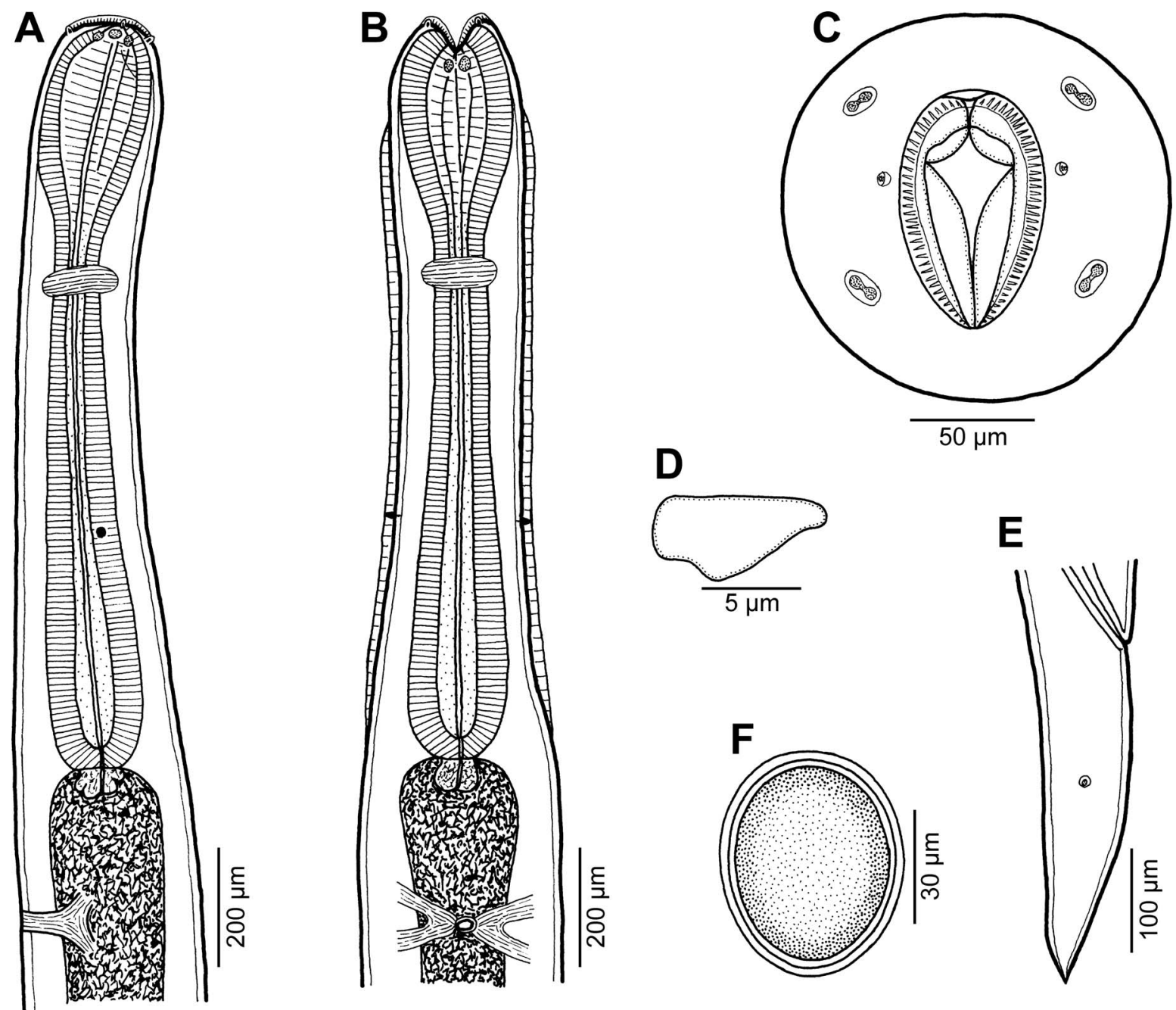

Figure 7. Cucullanus incognitus n. sp. from Dentex fourmanoiri, female. (A, B) Anterior end of body, lateral and ventral views; (C) cephalic end, apical view; (D) deirid, lateral view; (E) tail, lateral view; (F) egg.

\section{Cucullanus incognitus n. sp. Figures 7, 8}

urn:1sid:zoobank.org:act:C2C4E9A4-A750-4D94-961F1B9AEA3B79AF

Type host: Dentex fourmanoiri Akazaki et Séret (Sparidae, Perciformes) (total body length $242 \mathrm{~mm}$, weight $349 \mathrm{~g}$ ).

Site of infection: Digestive tract.

Type locality: Deep sea, external slope of the barrier reef,

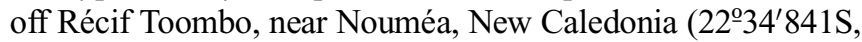
166-27'612E, depth 200-350 m) (collected 2 July 2009).

Prevalence and intensity: 1 fish infected/1 fish examined; 2 specimens.

Deposition of type specimens: Muséum national d'Histoire naturelle, Paris, France (female holotype, MNHN JNC 2992); Helminthological Collection, Institute of Parasitology, Biology Centre of the Czech Academy of Sciences, České Budějovice, Czech Republic (female paratype mounted on SEM stub, Cat. No. N-1169).

Etymology: The specific name incognitus (= unknown) is a Latin adjective and relates to the fact that this nematode species was previously unknown.

\section{Description}

Female (2 ovigerous specimens; holotype; measurements of paratype in parentheses): Medium-sized nematodes. Body whitish, elongate, slightly narrowed in region between posterior end of pseudobuccal capsule and posterior end of oesophagus (Figs. 7A, 7B, 8F, 8G); length of body 15.14 (13.38) mm, maximum width 354 (354); body length at level of oesophastome 272 (245), at middle of oesophagus 258 (231). Narrow lateral cervical alae present, 15 (15) wide, commencing approximately at level of mid-length of pseudobuccal capsule and extending posteriorly to short distance anterior to posterior end of oesophagus (Figs. 7B, 8D, 8F, 8G). Cephalic end slightly asymmetrical in lateral view (Figs. 7A, 8A). Oral aperture dorsoventrally elongate, surrounded by raised narrow membranous ala (collarette) supported by row of $c .90$ minute basal teeth (Figs. 7C, 8A-8C, 8E). Four submedian cephalic double papillae and pair of lateral amphids present (Figs. 7C, $8 \mathrm{~A}-8 \mathrm{C}, 8 \mathrm{~F}, 8 \mathrm{G})$. Oesophagus muscular, expanded at anterior end to form elongate pseudobuccal capsule (oesophastome), approximately twice as long as wide (Figs. 7A, 7B); length 

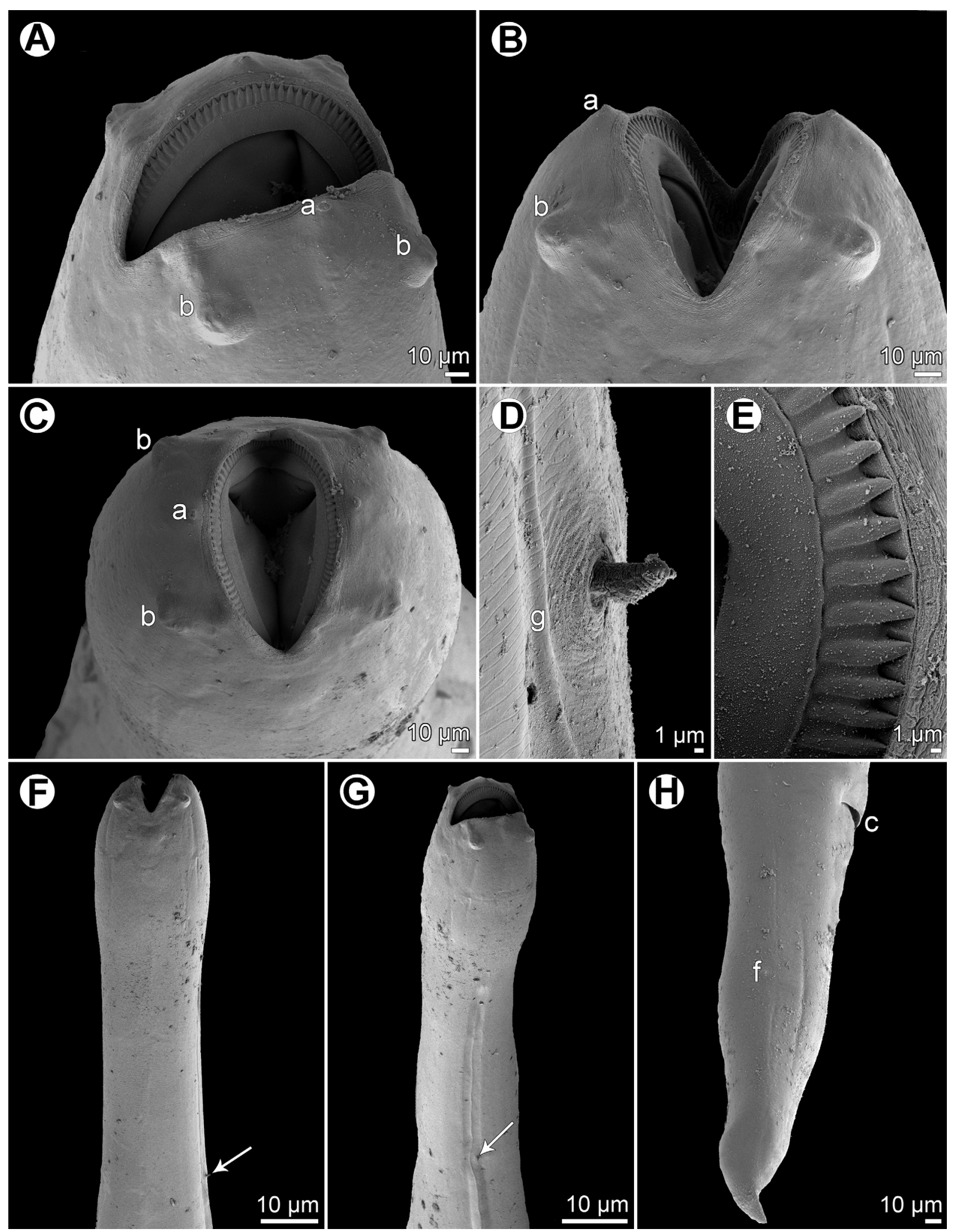

Figure 8. Cucullanus incognitus n. sp., scanning electron micrographs of female. (A, B) Cephalic end, sublateral and dorsoventral views, respectively; (C) same, apical view; (D) deirid and cervical ala, sublateral view; (E) cephalic teeth; (F, G) anterior end of body, dorsoventral and sublateral views, respectively (arrows indicate deirids; note cervical alae); (H) tail, lateral view. (a) amphid; (b) cephalic double papilla; (c) anus; (f) phasmid; (g) cervical ala. 
of oesophastome 394 (394), width 231 (204). Posterior part of oesophagus also expanded, somewhat narrower than oesophastome in lateral view (Figs. 7A, 7B); maximum width of posterior part of oesophagus 204 (177), minimum width of oesophagus in region of nerve ring 109 (82). Entire length of oesophagus including oesophastome 1.54 (1.40) $\mathrm{mm}$, representing $10(10) \%$ of total body length. Oesophagus opens into intestine through large valve. Nerve ring encircles oesophagus at distance of 503 (490) from anterior extremity, representing $33(35) \%$ of oesophageal length. Deirids situated approximately at mid-way between nerve ring and posterior end of oesophagus, at 952 (938) from anterior end of body (Figs. 7A, 7B, 8D, 8F, 8G). Postdeirids not found. Excretory pore at short distance posterior to oesophago-intestinal junction (Fig. 7A, 7B), at 1.71 (1.61) $\mathrm{mm}$ from anterior extremity. Vulva situated $9.14(8.16) \mathrm{mm}$ from anterior end of body, i.e. at $60(61) \%$ of body length; vulval lips not elevated. Vagina directed anteriorly from vulva. Eggs oval, thin-shelled, size 75-84 × 45-66 (81-90 × 54-60) (Fig. 7F). Tail conical, 394 (394) long, sharply pointed at tip, provided with pair of lateral phasmids located approximately at its middle (Figs. 7E, 8H). Male: Not known.

\section{Remarks}

Even though males of this new species are not known, C. incognitus n. sp. can be distinguished from the great majority of Cucullanus spp. by the presence of lateral cervical alae. Of the many species of Cucullanus, the presence of cervical alae, as found in C. incognitus, has hitherto been described only in C. truttae, a parasite mainly of freshwater salmonids (Salmonidae) in the Holarctic [21, 28], and in two recently established species parasitizing marine fishes in New Caledonian waters, i.e., C. epinepheli Moravec \& Justine, 2017 parasitic in Epinephelus chlorostigma (Valenciennes) (Serranidae, Perciformes) and C. austropacificus n. sp. from Conger cinereus reported in the present paper. Rasheed [38] mentioned the presence of asymmetrical "cuticular expansions in the form of cephalic alae" in C. theraponi Rasheed, 1968 from "Therapon" (= Terapon?) sp. (Terapontidae, Perciformes) and Hilsa sp. (Clupeidae, Clupeiformes) from off Pakistan, but these formations are different from cervical alae.

Cucullanus incognitus n. sp. differs from C. epinepheli in that its posterior portion of the oesophagus is narrower (vs. markedly wider) than the anterior oesophastome, its deirids are situated more anteriorly in relation to the length of the oesophagus (at $68 \%$ vs. $77-86 \%$ of oesophagus length) and the hosts belong to different fish families (Sparidae vs. Serranidae). In contrast to $C$. austropacificus, the gravid females of the new species are much smaller (body length approximately $13-15 \mathrm{~mm} v$ s. $26-35 \mathrm{~mm}$ ), their oesophastome is more elongate (approximately twice as long as wide vs. approximately as long as wide) and their eggs are smaller $(75-84 \times 45-66 \mu \mathrm{m} v s .84-108 \times 48-57 \mu \mathrm{m})$ and of a different shape (oval vs. elongate-oval); hosts of these two species belong to different fish orders (Perciformes vs. Anguilliformes). Regarding C. truttae, it can be easily distinguished from $C$. incognitus by the conspicuously asymmetrical cephalic end and by the excretory pore located at the mid-distance between the nerve ring and the oesophago-intestinal junction (vs. excretory pore posterior to the oesophageal end).

To date, the only nominal species of Cucullanus previously described from seabreams (Sparidae) are C. chrysophrydis Gendre, 1928, parasitizing Pagellus bogaraveo (Brünnich) and Sparus aurata Linnaeus off the Atlantic coast of Africa [7, 13, 43], and C. protrudens Pereira, Vieira \& Luque in Vieira et al., 2015, a parasite of Pagrus pagrus (Linnaeus) from off the Atlantic coast of Brazil [44]. Both these species differ from $C$. incognitus $\mathrm{n}$. sp. in the absence (vs. presence) of cervical alae and in the location of phasmids in the second half of the tail ( $v s$. at mid-length of tail). In addition, the vulva of C. protrudens is preequatorial, at $41 \%-43 \%$ of the body length (vs. postequatorial in the new species, at $60 \%-61 \%$ of body length). Moreover, all these three nematode species parasitize hosts belonging to different genera (Pagellus Valenciennes, Pagrus Cuvier and Sparus Linnaeus vs Dentex Cuvier) and they occur in geographically very distant regions (C. chrysophrydis and C. protrudens in the Atlantic Ocean vs C. incognitus n. sp. in the Pacific Ocean).

An unidentified species of Cucullanus, Cucullanus sp. from Pagrus sp., was reported by Vassiliadès [43] in the list of helminth parasites of marine fishes off the coast of Senegal (Atlantic Ocean). Cucullanus sp. was also reported from Pagrus auratus (Forster) in the Pacific Ocean, New Zealand [39]. However, in this case, specimens of another cucullanid genus Dichelyne Jägerskiöld, 1902 were probably misidentified as Cucullanus, as indicated by the small body measurements and accompanying illustrations (the intestinal caecum in Dichelyne spp. is sometimes difficult to observe and was probably overlooked by the authors). This is also supported by the fact that Cucullanellus (= Dichelyne) cnidoglanis Johnston \& Mawson, 1945 was reported from the same host species (P. auratus) in the same region (off New Zealand) [6]. Three species of the Sparidae, Acanthopagrus schlegelii (Bleeker), Dentex (reported as Evynnis Jordan \& Thompson) tumifrons (Temminck \& Schlegel) and Pagrus major (Temminck et Schlegel), were reported as hosts of Dichelyne jialaris Luo, Guo, Fang \& Huang, 2004 from off China and Japan [16, 29].

The authors are aware of the fact that the description of C. incognitus n. sp. is based solely on female morphology, a procedure that cannot generally be recommended; however, in this case, the new species appears to be well established and, therefore, we consider it useful to give the species a name rather than to report it as Cucullanus sp. and to wait years until conspecific males are available; the host is extremely rarely collected.

It should be noted that the only host specimen (D. fourmanoiri) examined harboured, in addition to $C$. incognitus n. sp., two specimens of the cystidicolid nematode Rasheedia heptacanthi Moravec \& Justine, 2018 in the digestive tract [25].

\section{Discussion}

As mentioned above, the morphology of the numerous species of Cucullanus is rather uniform. Therefore, the separation of similar species based solely on morphological features studied by LM may be problematic, especially in the situation when some Cucullanus spp. have been insufficiently described. Nevertheless, some papers published during last two decades 
(e.g. $[15,17,22-24,27,30,32-34,41,42,44])$ have shown the importance of the use of SEM for the taxonomy of these nematodes, because some features are difficult to observe or are not visible at all under the LM.

This concerns, for example, the exact number and distribution of caudal papillae in the male or the situation of deirids and the excretory pore. One such feature is the presence of narrow lateral cervical alae, observable in dorsoventral view, which can be easily overlooked when using LM, but their presence can be confirmed by SEM. The presence/absence of cervical alae appears to be an important specific taxonomic feature in Cucullanus; as stated above, of many described species of Cucullanus, cervical alae have hitherto been reported only in C. austropacificus n. sp., C. epinepheli, C. incognitus n. sp. and $C$. truttae. According to experimental observations [19], cervical alae of third- and fourth-stage larvae of C. truttae are much wider than those of conspecific adults.

As revealed by SEM, taxonomically very important features are found in the structures of the cloacal region in the male. It seems that many species of Cucullanus possess a small, rounded median precloacal elevation, sometimes reported as the median precloacal papilla or the median precloacal organ; in fact, such an elevation usually bears a single minute papilla (e.g. in C. gymnothoracis n. sp., see Fig. 6D) or, less often, two minute papillae (C. epinepheli, C. genypteri Sardella, Navone $\&$ Timi, 1997) are visible on its surface [44], present paper. However, there are species of Cucullanus (e.g. C. bulbosus), in which the median precloacal elevation is lacking [22].

In species of Cucullanus possessing the median precloacal elevation, the elevation may be connected with the conspicuously large, flat posterior outgrowth of the anterior cloacal lip that covers the cloacal aperture (in $C$. epinepheli) or there is a median, ventrally oriented outgrowth protruding from the base of the anterior cloacal lip; this may be rounded and small (e.g. in C. mycteropercae Mejía-Madrid \& GuillénHernández, 2011, C. pseudopercis Pereira, Vieira \& Luque in Vieira et al., 2015 or C. oceaniensis Moravec, Sasal, Würtz \& Taraschewski, 2005) or large (e.g. in C. pedroi or C. austropacificus n. sp., see Fig. 3F), or the outgrowth forms a narrow, slightly protruding transverse plate adherent to the anterior cloacal lip in ventral view, as visible in C. gymnothoracis $\mathrm{n}$. sp. (Fig. 6D; [17, 22, 30, 44], present paper). The posterior cloacal lip may be conspicuously elevated in some Cucullanus spp. (e.g. C. costaricensis López-Caballero, Osorio-Sarabia \& García-Prieto, 2009) or may be provided with a large, markedly elevating transverse outgrowth ( $C$. pedroi, C. austropacificus n. sp., see Fig. 3C) [15, 44], present paper.

Consequently, when studying cucullanid species, as well as other nematodes, it is highly desirable to examine them by both LM and SEM. Of course, the use of molecular methods, if possible, is also important.

\section{Key to species of Cucullanus parasitic in anguilliform fishes (Anguilliformes):}

1. Mouth markedly inclined dorsally. Cuticular lining of oesophastome consisting of complex set of thickened cuticularized pieces separated by sutures. Narrow cervical alae present. Male with ventral sucker and spicules 345-775 $\mu \mathrm{m}$ long. Parasitic mainly in Holarctic freshwater Salmonidae; in Europe also in Anguilla anguilla (Anguillidae) serving as postcyclic host ............. subgenus Truttaedacnitis

\section{C. truttae}

- Mouth slightly inclined dorsally. Cuticularized plates of oesophastome few in number and separated by simple Y-shaped suture. Cervical alae mostly absent, ventral sucker usually present .......... subgenus Cucullanus ............................................................. 2

2. Ventral sucker absent. Spicules 636-924 $\mu \mathrm{m}$ long, length of gubernaculum 156-204 $\mu \mathrm{m}$. Parasitic in Muraenesox cinereus (Muraenesocidae) in the East China Sea (off China) ........................................................... . wangi

- Ventral sucker present ............................................ 3

3. Spicules shorter than $500 \mu \mathrm{m}$..................................... 4

- Spicules at least $590 \mu \mathrm{m}$ long ................................ 5

4. First pair of preanal papillae located short distance anterior to ventral sucker. Oesophastome elongate, longer than wide. Spicules $440 \mu \mathrm{m}$ long, length of gubernaculum $90 \mu \mathrm{m}$. Parasitic in Muraena robusta (Muraenidae) off the Atlantic coast of Africa (Senegal)

\section{C. murenophidis}

- First pair of preanal papillae at level of ventral sucker. Oesophastome approximately as long as wide. Spicules $430 \mu \mathrm{m}$ long, length of gubernaculum $140 \mu \mathrm{m}$. Parasitic in Muraenesox cinereus (Muraenesocidae) off Japan

\section{C. muraenesocis}

5. First pair of preanal papillae at level of ventral sucker (near its anterior end). Spicules at most $800 \mu \mathrm{m}$ long

- First pair of preanal papillae located short distance anterior to ventral sucker. Spicules usually longer than $800 \mu \mathrm{m}$ 8

6. Oesophastome elongate, its length approximately one and half of its width. Length of spicules $800 \mu \mathrm{m}$. Parasitic in Anguilla japonica (Anguillidae) in Taiwan Strait (off China) ............................................ C. anguillae

- Oesophastome approximately as long as wide. Spicules shorter than $800 \mu \mathrm{m}$.................................... 7

7. Length of spicules 590-650 $\mu \mathrm{m}$. Parasitic in Anguilla anguilla (Anguillidae) on the coast of the Red Sea (Gulf of Suez, Egypt) ........... C. egyptae [species inquirenda]

- Length of spicules 637-760 $\mu \mathrm{m}$. Parasitic in Muraenichthys gymnopterus (Ophichthidae) in the South China Sea (off China) C. hainanensis

8. Deirids located somewhat posterior to nerve ring. Spicules 990-1,200 $\mu \mathrm{m}$ long. Excretory pore in region of deirids. Parasitic in Gymnothorax pictus and G. javanicus (Muraenidae) in the South Pacific (off Australia and French Polynesia)

C. australiensis

- Deirids located at mid-length of oesophagus or anterior to oesophago-intestinal junction. Excretory pore slightly anterior, at level or posterior to end of oesophagus 9

9. Oesophastome elongate, its length approximately one and half of its width 
- Oesophastome approximately as long as wide 12

10. Oesophastome approximately as wide as posterior part of oesophagus. Spicules $600-1,260 \mu \mathrm{m}$ long. Parasitic in Conger conger (Congridae) (type host) and, allegedly, Muraena helena (Muraenidae) off the Atlantic coast of Europe and Africa C. hians

- Oesophastome wider than posterior part of oesophagus 11

11. Deirids located at posterior third of oesophagus. Median outgrowth adherent to inner rim of anterior cloacal lip as large as median precloacal papilla-like formation. Posterior cloacal lip with large, conspicuously elevated transverse outgrowth. Spicules 900-1,600 $\mu \mathrm{m}$ long, with conspicuously broad alae. Body length of male approximately 10-16 mm, that of gravid female 13-18 mm. Parasitic in Conger orbignianus (Congridae) off the Atlantic coast of Argentina

C. pedroi

- Deirids just anterior to oesophago-intestinal junction. Median outgrowth adherent to inner rim of anterior cloacal lip distinctly smaller than median precloacal papilla-like formation. Posterior cloacal lip without conspicuously elevated outgrowth. Spicules 668-1020 $\mu \mathrm{m}$, spicular alae not conspicuously broad. Body length of male approximately 6-10 $\mathrm{mm}$, that of gravid female 9-14 $\mathrm{mm}$. Parasitic in Anguilla marmorata (type host) and A. cf. obscura (Anguillidae) in Polynesia and Melanesia (Futuna and Fiji Islands)

C. oceaniensis

12. Narrow cervical alae absent. Anterior and posterior cloacal lips without conspicuously elevated median outgrowths 13

- Narrow cervical alae present. Anterior and posterior cloacal lips each with conspicuously elevated median outgrowth. Gubernaculum 225-291 $\mu \mathrm{m}$ long. Length of spicules $1.30-1.65 \mathrm{~mm}$. Body length of male approximately 16-26 mm, that of female 26-35 mm. Parasitic in Conger cinereus (Congridae) in the South Pacific (off New Caledonia)

C.austropacificus n. sp.

13. Deirids located at mid-length of oesophagus. Excretory pore slightly anterior to or at level of oesophago-intestinal junction. Phasmids of male situated between two last pairs of subventral postanal papillae. Gubernaculum 108-180 $\mu \mathrm{m}$ long. Length of spicules $980-1,600 \mu \mathrm{m}$. Body length of male approximately $7-18 \mathrm{~mm}$, that of female 14-26 mm. Parasitic in Conger myriaster (Congridae) off Japan and Korea ................. C. robustus

- Deirids somewhat anterior to end of oesophagus. Excretory pore short distance posterior to oesophago-intestinal junction. Phasmids of male situated anterior to last two pairs of subventral postanal papillae. Gubernaculum $201 \mu \mathrm{m}$ long. Length of spicules $1116 \mu \mathrm{m}$. Body length of male $15 \mathrm{~mm}$. Parasitic in Gymnothorax chilospilus (Muraenidae) in the South Pacific (off New Caledonia). C. gymnothoracis n. sp.

Acknowledgements. We wish to thank colleagues and students who participated in the parasitological survey in New Caledonia: Adeline Grugeaud, Marine Briand and Eva Řehulková. Xavier
Neyrat (Aquarium des Lagons, Nouméa) collected the conger. The identification of fishes was confirmed, from photographs, by Bernard Séret (MNHN, Paris). Thanks are also due to the Laboratory of Electron Microscopy, Institute of Parasitology, Biology Centre CAS, institution supported by the MEYS CR (LM2015062 Czech-BioImaging) for their support with obtaining scientific data presented in this paper, and to Blanka Škoríková of the same Institute for help with the illustrations. This study was also partly supported by the Czech Science Foundation (Grant. No. P505/ 12G112) and by institutional support (RVO:60077344, Institute of Parasitology, BC, CAS).

\section{Conflict of interest}

The Editor-in-Chief of Parasite is one of the authors of this manuscript. COPE (Committee on Publication Ethics, https:// publicationethics.org), to which Parasite adheres, advises special treatment in these cases. In this case, the peer-review process was handled by an Invited Editor, Jérôme Depaquit.

\section{References}

1. Abdel-Ghaffar F, Bashtar A-R, Abdel-Gaber R, Morsy K, Mehlhorn H, Al Quraishy S, Mohammed S. 2014. Cucullanus egyptae sp. nov. (Nematoda, Cucullanidae) infecting the European eel Anguilla anguilla in Egypt. Morphological and molecular phylogenetic studies. Parasitology Research, 113, 3457-3465.

2. Baylis HA. 1927. Some new parasitic nematodes from Australia. Annals and Magazine of Natural History, Series, 9(20), 214-225.

3. Beveridge I, Bray RA, Cribb TH, Justine J-L. 2014. Diversity of trypanorhynch metacestodes in teleost fishes from coral reefs off eastern Australia and New Caledonia. Parasite, 21, 60.

4. Bray RA, Justine J-L. 2011. Acanthocolpidae (Digenea) of marine fishes off New Caledonia, with the descriptions of two new species. Folia Parasitologica, 58, 35-47.

5. Briand MJ, Letourneur Y, Bonnet X, Wafo E, Fauvel T, Brischoux F, Guillou G, Bustamante P. 2014. Spatial variability of metallic and organic contamination of anguilliform fish in New Caledonia. Environmental Science and Pollution Research, 21, 4576-4591.

6. Brunsdon RV. 1953. A systematic study of the nematodes parasitic in New Zealand, with an account of the life history and culture of Stomachus marinus (Linn., 1776). M.Sc. thesis, Victoria University of Wellington. (Ex Sharples \& Evans 1995).

7. Campana-Rouget Y. 1957. Parasites de poissons de mer ouestafricains récoltés par J. Cadenat. Nématodes $\left(4^{\mathrm{e}}\right.$ note $)$. Sur quelques espèces de Cucullanidae. Révision de la sous-famille. Bulletin de l'Institut Fondamental d'Afrique Noire, Série A, 19, 417-465.

8. Campana-Rouget Y, Chabaud A-G. 1956. Helminthes des environs de Banyuls III. Sur trois espèces de Cucullanus (Camallanoidea, Nematoda) parasites des poisons. Vie et Milieu, 7, 267-279.

9. Daniel VI, Timi JT, Sardella NH. 2002. Cucullanus marplatensis sp. n. (Nematoda, Cucullanidae) parasitizing Odontesthes argentinensis (Valenciennes, 1835) (Pisces, Atherinidae) from Argentinian waters. Acta Parasitologica, 47, 41-46.

10. Dujardin F. 1845. Histoire naturelle des helminthes ou vers intestinaux. Paris, XVI $+654+15$ pp. 
11. Froese R, Pauly D, Editors, 2017. FishBase. World Wide Web electronic publication, http://www.fishbase.org, 07/2017.

12. González-Solís D, Tuz-Paredes VM, Quintal-Loria MA. 2007. Cucullanus pargus sp. n. (Nematoda: Cucullanidae) from the grey snapper Lutjanus griseus off the southern coast of Quintana Roo. Mexico. Folia Parasitologica, 54, 220-224.

13. Ivashkin VM, Khromova LA. 1976. Cucullanata and Gnathostomatata of animals and man and the diseases caused by them. Osnovy Nematodologii 27. Nauka, Moscow, 388 pp. (In Russian)

14. Justine J-L, Briand MJ, Bray RA. 2012. A quick and simple method, usable in the field, for collecting parasites in suitable condition for both morphological and molecular studies. Parasitology Research, 111, 341-351.

15. López-Caballero J, Osorio-Sarabia D, García-Prieto L. 2009. Cucullanus costaricensis n. sp. (Nematoda: Cucullanidae), a parasite of Bagre pinnimaculatus (Siluriformes: Ariidae) from Río Tempisque, Costa Rica. Journal of Parasitology, 95, 413423.

16. Luo D, Guo S, Fang W, Huang H. 2004. Observations on a cucullanid nematode of marine fishes from Taiwan Strait, Dichelyne (Cucullanellus) jialaris n. sp. Journal of Parasitology, 90, 608-611.

17. Mejía-Madrid HH, Guillén-Hernández SG. 2011. A new cucullanid from the black grouper Mycteroperca bonaci (Osteichthyes: Serranidae) off the coast of Yucatán, México. Journal of Parasitology, 97, 122-127.

18. Morand S, Rigby MC. 1998. Cucullanin nematodes from coral reef fishes of French Polynesia, with a description of Cucullanus faliexae n. sp. (Nematoda: Chitwoodchabaudiidae). Journal of Parasitology, 84, 1213-1217.

19. Moravec F. 1979. Observations on the development of Cucullanus (Truttaedacnitis) truttae (Fabricius, 1794) (Nematoda: Cucullanidae). Folia Parasitologica, 26, 295-307.

20. Moravec F. 2003. Observations on the metazoan parasites of the Atlantic salmon (Salmo salar) after its reintroduction into the Elbe River basin in the Czech Republic. Folia Parasitologica, 50, 298-304.

21. Moravec F. 2013. Parasitic nematodes of freshwater fishes of Europe. Revised second edition, Prague: Academia, $601 \mathrm{pp}$.

22. Moravec F, Gey D, Justine J-L. 2016. Nematode parasites of four species of Carangoides (Osteichthyes: Carangidae) in New Caledonian waters, with a description of Philometra dispar $\mathrm{n}$. sp. (Philometridae). Parasite, 23, 40.

23. Moravec F, Justine J-L. 2011. Cucullanid nematodes (Nematoda: Cucullanidae) from deep-sea marine fishes off New Caledonia, including Dichelyne etelidis n. sp. Systematic Parasitology, 78, 95-108.

24. Moravec F, Justine J-L. 2017. Two new species of nematode parasites, Cucullanus epinepheli sp. n. (Cucullanidae) and Procamallanus (Spirocamallanus) sinespinis sp. n. (Camallanidae), from marine serranid and haemulid fishes off New Caledonia. Folia Parasitologica, 64, 011.

25. Moravec F, Justine J-L. 2018. Rasheedia n. nom. (Nematoda: Physalopteridae) for Bulbocephalus Rasheed, 1966 (a homonym of Bulbocephalus Watson, 1916), with description of Rasheedia heptacanthi $\mathrm{n}$. $\mathrm{sp}$. and $R$. novaecaledoniensis $\mathrm{n} . \mathrm{sp}$. from perciform fishes off New Caledonia. Parasite, 25, 39.

26. Moravec F, Kohn A, Fernandes BMM. 1997. New observations on seuratoid nematodes parasitic in fishes of the Paraná River, Brazil. Folia Parasitologica, 44, 209-223.

27. Moravec F, Lorber J, Konečný R. 2008. Cucullanus maldivensis n. sp. (Nematoda: Cucullanidae) and some other adult nematodes from marine fishes off the Maldive Islands. Systematic Parasitology, 70, 61-69.
28. Moravec F, Malmqvist B. 1977. Records of Cucullanus truttae (Fabricius, 1794) (Nematoda: Cucullanidae) from Swedish brook lampreys, Lampetra planeri (Bloch). Folia Parasitologica, 24, 323-329.

29. Moravec F, Nagasawa K, Madinabeitia I. 2018. Redescription of Dichelyne (Cucullanellus) jialaris (Nematoda: Cucullanidae), a parasite of seabreams (Perciformes: Sparidae) in East Asia. Acta Parasitologica, DOI: 10.1515/ap-2018-00.

30. Moravec F, Sasal P, Würtz J, Taraschewski H. 2005. Cucullanus oceaniensis sp. n. (Nematoda: Cucullanidae) from Pacific eels (Anguilla spp.). Folia Parasitologica, 52, 343-348.

31. Moravec F, Scholz T. 2015. Macroparasites and their communities of the European eel Anguilla anguilla (Linnaeus) in the Czech Republic. Folia Parasitologica, 62, 033.

32. Park J-K, Moravec F. 2008. Redescription of Cucullanus robustus (Nematoda: Cucullanidae) from the conger eel Conger myriaster off Korea. Zootaxa, 1729, 1-7.

33. Pereira FB, Vieira FM, Luque JL. 2014. A new species of Cucullanus Müller, 1777 (Nematoda: Cucullanidae) parasitic in the grey triggerfish Balistes capriscus Gmelin (Osteichthyes: Balistidae) off Rio de Janeiro, Brazil. Systematic Parasitology, 87, 283-291.

34. Pereira FB, Vieira FM, Luque JL. 2015. New morphological data and first description of gravid female of Cucullanus bagre Petter, 1974 (Seuratoidea: Cucullanidae) from Bagre bagre (Linnaeus, 1766) (Siluriformes: Ariidae) off Brazil. Acta Parasitologica, 60, 138-145.

35. Petter A-J. 1974. Essai de classification de la famille des Cucullanidae. Bulletin du Muséum National d'Histoire Naturelle, 3 série No. 255, Zoologie, 177, 1469-1490.

36. Petter A-J, Le Bel J. 1992. Two new species in the genus Cucullanus (Nematoda - Cucullanidae) from the Australian region. Memórias do Instituto Oswaldo Cruz, 87(Supplement 1), 201-206.

37. Petter A-J, Radujković BM. 1989. Parasites des poissons marins du Montenegro: Nematodes. Acta Adriatica, 30, 195-236.

38. Rasheed S. 1968. The nematodes of the genus Cucullanus Mueller, 1777 from the marine fish of Karachi coast. Anales de la Escuela Nacional de Ciencias Biológicas, 15, 23-59.

39. Sharples AD, Evans CW. 1995. Taxonomy of the metazoan parasites of the snapper Pagrus auratus in New Zealand 2. Endoparasites. New Zealand Journal of Zoology, 22, 163-174.

40. Timi JT, Lanfranchi AL. 2006. A new species of Cucullanus (Nematoda: Cucullanidae) parasitizing Conger orbignianus (Pisces: Congridae) from Argentinean waters. Journal of Parasitology, 92, 151-154.

41. Xu Z, Zhang L-P, Li L. 2014. Morphological and molecular characterization of Cucullanus hainanensis sp. nov. (Ascaridida: Cucullanidae) from Muraenichthys gymnopterus (Bleeker) (Anguilliformes: Ophichthidae) in the South China Sea. Acta Parasitologica, 59, 710-716.

42. Xu Z, Chen H-X, Ju H-D, Li L. 2017. Morphological and molecular characterization of Cucullanus bourdini Petter et Le bel, 1992 (Ascaridida: Cucullanidae) from the yellowback fusilier Caesio xanthonota Bleeker (Perciformes: Caesionidae). Acta Parasitologica, 62, 199-206.

43. Vassiliadès G. 1982. Helminthes parasites des poisons de mer des côtes du Sénégal. Bulletin de l'Institut Fondamental d'Afrique Noire, Série A, 44, 78-99.

44. Vieira FM, Pereira FB, Pantoja C, Soares IA, Pereira AN, Timi JT, Scholz T, Luque JL. 2015. A survey of nematodes of the genus Cucullanus Müller, 1777 (Nematoda, Seuratoidea) parasitic in marine fishes off Brazil, including description of three new species. Zootaxa, 4039, 289-311. 
45. Wang P, Ling X. 1975. Some nematodes of the suborder Camallanata from Fujian Province, with notes on their life histories. Acta Zoologica Sinica, 21, 350-358 (In Chinese with English summary).

46. Yamaguti S. 1935. Studies on the helminth fauna of Japan. Part 9. Nematodes of fishes, 1. Japanese Journal of Zoology, 6, 337-386.
47. Yamaguti S. 1961. Studies on the helminth fauna of Japan. Part 57. Nematodes of fishes, III. Journal of Helminthology, R. T. Leiper Supplement, 217-228.

48. Yin W-Z, Zhang N-X. 1983. On two new species of parasitic nematodes from marine fishes from Zhoushan Islands, China. Acta Zootaxonomica Sinica, 8, 7-10.

Cite this article as: Moravec F \& Justine J-L. 2018. Three new species of Cucullanus (Nematoda: Cucullanidae) from marine fishes off New Caledonia, with a key to species of Cucullanus from Anguilliformes. Parasite 25, 51.

\section{๑) PARASTE}

An international open-access, peer-reviewed, online journal publishing high quality papers on all aspects of human and animal parasitology

Reviews, articles and short notes may be submitted. Fields include, but are not limited to: general, medical and veterinary parasitology; morphology, including ultrastructure; parasite systematics, including entomology, acarology, helminthology and protistology, and molecular analyses; molecular biology and biochemistry; immunology of parasitic diseases; host-parasite relationships; ecology and life history of parasites; epidemiology; therapeutics; new diagnostic tools.

All papers in Parasite are published in English. Manuscripts should have a broad interest and must not have been published or submitted elsewhere. No limit is imposed on the length of manuscripts.

Parasite (open-access) continues Parasite (print and online editions, 1994-2012) and Annales de Parasitologie Humaine et Comparée (1923-1993) and is the official journal of the Société Française de Parasitologie. http://parasite.edmgr.com/ 